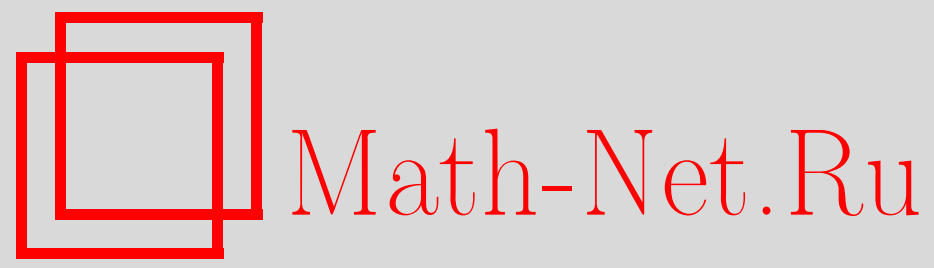

О. Л. Бозиев, Приближенное решение нагруженного гиперболического уравнения с однородными начальными условиями, Вестник ТвГУ. Серия: Прикладная математика, 2017, выпуск 2, 49-58

DOI: https://doi.org/10.26456/vtpmk108

Использование Общероссийского математического портала Math-Net.Ru подразумевает, что вы прочитали и согласны с пользовательским соглашением

http: //www.mathnet.ru/rus/agreement

Параметры загрузки:

IP: 3.80 .253 .173

26 апреля 2023 г., 15:32:35 
УДК 517.956.35

\section{ПРИБЛИЖЕННОЕ РЕШЕНИЕ НАГРУЖЕННОГО ГИПЕРБОЛИЧЕСКОГО УРАВНЕНИЯ С ОДНОРОДНЫМИ НАЧАЛЬНЫМИ УСЛОВИЯМИ}

\section{Бозиев О.Л.}

Институт информатики и проблем регионального управления Кабардино-Балкарского научного центра РАН, г. Нальчик

Поступила в редакиию 04.02.2017, после переработки 31.03.201\%.

Предлагается метод решения смешанной задачи с однородными начальными условиями для нагруженного гиперболического уравнения, содержащего интеграл натуральной степени модуля неизвестной функции. Приближенное решение ищется с помощью априорных оценок решения поставленной задачи. Получена формула, выражающая это решение через решение обыкновенного дифференциального уравнения, ассоциированного с исходным нагруженным уравнением.

Ключевые слова: нелинейные уравнения в частных производных, нагруженные уравнения в частных производных, априорные оценки, приближенные решения.

Вестник ТвГУ. Серия: Прикладная математика. 2017. № 2. С. 49-58.

\section{Введение}

Нелинейное уравнение вида

$$
u_{t t}-a^{2} u_{x x}+b|u|^{p} u_{t}=0,
$$

с положительными параметрами $a$ и $b$, натуральным $p$ и начально-краевыми условиями различного вида в прямоугольной области исследовалось в [1] и многих других работах в качестве модели некоторых нестационарных процессов. Его модификация

$$
u_{t t}-a^{2} u_{x x}+b u_{t} \int_{\Omega}|u|^{p} d x=0
$$

является нагруженным уравнением и может рассматриваться как аппроксимирующее по отношению к (1).

Уравнения вида (2) и его обобщения рассмотрены, например, в $[2,3]$. В этих и других работах доказаны теоремы существования и единственности обобщенных решений соответствующих краевых задач.

Переход от (1) к (2) приводит к «ослаблению» нелинейности исходного уравнения без значительного искажения сути моделируемого процесса. В то же время это позволяет находить приближенное решения уравнения (1) путем нахождения 
точного или приближенного решения начально-краевой задачи для нагруженного уравнения (2), которое затем принимается за приближенное решение исходного нелинейного уравнения.

С применением такого подхода в $[4,5]$ получены формулы общих членов последовательностей приближенных решений начально-краевых задач для некоторых нагруженных уравнений, аппроксимирующих исходные нелинейные уравнения.

В [6] предложен способ нахождения приближенного решения первой смешанной задачи с однородными граничными условиями для уравнения (2), использующий априорные оценки решения поставленной задачи.

В данной работе для уравнения (2) этим же способом ищется решение смешанной задачи с однородными начальными условиями.

\section{1. Априорные оценки}

В области $Q=\{(x, t): 0<x<l, 0<t<T\}$ при натуральном $p>3$ рассмотрим уравнение (2). Требуется найти интегрируемую функцию $u(x, t) \in C^{2,2}(\bar{Q})$, удовлетворяющую уравнению (2) в области $Q$, а также условиям

$$
\begin{gathered}
u(x, 0)=0, u_{t}(x, 0)=0,0 \leqslant x \leqslant l, \\
u(0, t)=\psi_{1}(t), u(l, t)=\psi_{2}(t), 0 \leqslant t \leqslant T,
\end{gathered}
$$

с функциями $\psi_{1}(t), \psi_{2}(t) \in C^{1}(0, T)$.

Установим некоторые априорные оценки решения задачи (2)-(4), необходимые для нахождения ее приближенного решения.

Умножая (2) скалярно на $u_{t}$ с помощью стандартных для подобных случаев преобразований получаем следующие неравенства, выполняющиеся для всех значений $t \in[0, T]$ :

$$
\int_{\Omega}\left(u_{t}^{2}+a^{2} u_{x}^{2}\right) d x \leqslant C_{1}, \quad\left\|u_{t}\right\|_{2, \Omega}^{2} \leqslant C_{1}, \quad\left\|u_{x}\right\|_{2, \Omega}^{2} \leqslant \frac{C_{1}}{a^{2}}
$$

Здесь и далее равенством

$$
\|v\|_{p, \Omega}^{p}=\int_{\Omega}|v|^{p} d x
$$

выражается норма функции $v(t)$ в пространстве $L_{p}(\Omega), \Omega=[0, l]$.

Теорема 1. Пусть функция $u \in L_{p-2}(\Omega)$ является решением задачи (2)-(4), $а$ неубавающие функиии $\psi_{1}(t), \psi_{2}(t) \in L_{p-1}[0, T]$. Тогда функиия $\|u\|_{p, \Omega}^{p}$ ограничена константой, зависящей только от $t$.

Доказательство. Умножим уравнение (2) скалярно на функцию $u^{p-1}$

$$
\left(u_{t t}, u^{p-1}\right)-a^{2}\left(u_{x x}, u^{p-1}\right)+b \int_{\Omega}|u|^{p} d x\left(u_{t}, u^{p-1}\right)=0 .
$$


Преобразуем по отдельности каждое слагаемое:

$$
\begin{gathered}
\left(u_{t t}, u^{p-1}\right)=\frac{1}{p} \frac{d^{2}}{d t^{2}} \int_{\Omega} u^{p} d x-(p-1) \int_{\Omega} u_{t}^{2} u^{p-2} d x \\
\left(u_{x x}, u^{p-1}\right)=u_{x}(l, t) \psi_{2}^{p-1}(t)-u_{x}(0, t) \psi_{1}^{p-1}(t)-(p-1) \int_{\Omega} u_{x}^{2} u^{p-2} d x \\
\int_{\Omega}|u|^{p} d x\left(u_{t}, u^{p-1}\right)=\frac{1}{p} \int_{\Omega}|u|^{p} d x \cdot \frac{d}{d t} \int_{\Omega} u^{p} d x
\end{gathered}
$$

Возвращаясь к (6) и умножая его на $\operatorname{sgn}^{p} u$, приходим к уравнению

$$
\begin{gathered}
\frac{d^{2}}{d t^{2}} \int_{\Omega}|u|^{p} d x+\frac{b}{2} \frac{d}{d t}\left(\int_{\Omega}|u|^{p} d x\right)^{2}=p(p-1) \int_{\Omega}|u|^{p-2}\left(u_{t}^{2}-a^{2} u_{x}^{2}\right) d x+F_{1}(t), \\
F_{1}(t)=p a^{2}\left(u_{x}(l, t) \psi_{2}^{p-1}(t)-u_{x}(0, t) \psi_{1}^{p-1}(t)\right) \operatorname{sgn}^{p} u
\end{gathered}
$$

после интегрирования которого по $t$ с учетом однородности начальных условий получаем

$$
\begin{aligned}
\frac{d}{d t} \int_{\Omega}|u|^{p} d x+\frac{b}{2}\left(\int_{\Omega}|u|^{p} d x\right)^{2} & = \\
& =p(p-1) \int_{0}^{t} \int_{\Omega}|u|^{p-2}\left(u_{t}^{2}-a^{2} u_{x}^{2}\right) d x d t+\int_{0}^{t} F_{1}(t) d t
\end{aligned}
$$

K первому слагаемому в правой части (7) применим неравенство Гёльдера:

$$
\begin{aligned}
& \int_{0}^{t} \int_{\Omega}|u|^{p-2}\left(u_{t}^{2}-a^{2} u_{x}^{2}\right) d x d t \leqslant \\
& \quad \leqslant\left(\left.\left.\int_{0}^{t}\left|\int_{\Omega}\right| u\right|^{p-2} d x\right|^{2} d t\right)^{\frac{1}{2}}\left(\int_{0}^{t}\left|\int_{\Omega}\right| u_{t}^{2}-a^{2} u_{x}^{2}|d x|^{2} d t\right)^{\frac{1}{2}}
\end{aligned}
$$

Сомножители правой части полученного неравенства ограничены: первый в силу $u \in L_{p-2}(\Omega)$ :

$$
\left(\left.\left.\int_{0}^{t}\left|\int_{\Omega}\right| u\right|^{p-2} d x\right|^{2} d t\right)^{\frac{1}{2}} \leqslant\left(\int_{0}^{t}\left|C_{2}\right|^{2} d t\right)^{\frac{1}{2}} \leqslant \sqrt{t} C_{2}
$$

а второй - в силу первой из оценок (5):

$$
\left(\int_{0}^{t}\left|\int_{\Omega}\right| u_{t}^{2}-a^{2} u_{x}^{2}|d x|^{2} d t\right)^{\frac{1}{2}} \leqslant\left(\int_{0}^{t}\left|\int_{\Omega}\right| u_{t}^{2}+a^{2} u_{x}^{2}|d x|^{2} d t\right)^{\frac{1}{2}} \leqslant \sqrt{t} C_{1} .
$$

Следовательно,

$$
\int_{0}^{t} \int_{\Omega}|u|^{p-2}\left(u_{t}^{2}-a^{2} u_{x}^{2}\right) d x d t \leqslant C_{1} C_{2} t
$$


что позволяет перейти от уравнения (7) к неравенству

$$
\frac{d}{d t} \int_{\Omega}|u|^{p} d x+\frac{b}{2}\left(\int_{\Omega}|u|^{p} d x\right)^{2} \leqslant p(p-1) C_{1} C_{2} t+\int_{0}^{t}\left|F_{1}(t)\right| d t .
$$

Используя свойства функций $\psi_{1}(t)$ и $\psi_{2}(t)$, можно убедиться в том, что

$$
\begin{aligned}
\int_{0}^{t}\left|F_{1}(t)\right| d t \leqslant p a^{2}\left(\int_{0}^{t}\left|u_{x}(l, t)\right|\right. & \left.\left|\psi_{2}^{p-1}(t)\right| d t+\int_{0}^{t}\left|u_{x}(0, t)\right|\left|\psi_{1}^{p-1}(t)\right| d t\right) \leqslant \\
& \leqslant p a^{2}\left(\int_{0}^{T}\left|\psi_{2}(t)\right|^{p-1} d t+\int_{0}^{T}\left|\psi_{1}(t)\right|^{p-1} d t\right) C_{3}
\end{aligned}
$$

где

$$
C_{3}=\max \left\{\max _{t \in[0, T]}\left|u_{x}(l, t)\right|, \max _{t \in[0, T]}\left|u_{x}(0, t)\right|\right\} .
$$

С учетом этого проинтегрируем (8) и получим соотношение

$$
\int_{\Omega}|u|^{p} d x+\frac{b}{2} \int_{0}^{t}\left(\int_{\Omega}|u|^{p} d x\right)^{2} d t \leqslant \int_{\Omega}|u(x, 0)|^{p} d x+F(t),
$$

в котором первое слагаемое правой части равно нулю в силу первого условия (3), а

$$
F(t)=\frac{1}{2} p(p-1) C_{1} C_{2} t^{2}+p a^{2}\left(\int_{0}^{T}\left|\psi_{2}(t)\right|^{p-1} d t+\int_{0}^{T}\left|\psi_{1}(t)\right|^{p-1} d t\right) C_{3} t .
$$

Заметим, что $F(t) \leqslant F(T)$, в силу чего перейдем от $(9)$ к неравенству

$$
\|u\|_{2, \Omega}^{p} \leqslant \frac{b}{2} \int_{0}^{t}\left(\|u\|_{2, \Omega}^{p}\right)^{2} d t+F(T)
$$

Применяя к нему следствие из леммы Бихари [7], устанавливаем оценку

$$
\|u\|_{p, \Omega}^{p} \leqslant K(t)
$$

с правой частью

$$
K(t)=\frac{2 F(T)}{2-F(T) b t},
$$

выполняющуюся для всех $t \in[0, T], T<2 /(b F(T))$.

Таким образом, теорема доказана.

\section{2. Приближенное решение}

Для нахождения приближенного решения задачи (2)-(4) перейдем от (2) к ассоциированному с ним обыкновенному дифференциальному уравнению посредством процедуры, описанной в [6]. Она заключается в интегрировании (2) по пространственной переменной, применении теоремы о среднем значении интеграла, 
повторном интегрировании по $x$ и использования граничных условий (4) для определения произвольных функций, возникающих при интегрировании уравнения. В итоге приходим к соотношению

$$
u(x, t)=\frac{x}{2 a^{2}}\left(\frac{x}{l}-1\right)\left(\bar{u}^{\prime \prime}+b \bar{u}^{\prime}\|u\|_{p, \Omega}^{p}\right)+x \frac{\psi_{2}-\psi_{1}}{l}+\psi_{1},
$$

в котором

$$
\bar{u}(t)=\int_{\Omega} u d x .
$$

Применим преобразование (14) к функции (13) для того, чтобы перейти к обыкновенному дифференциальному уравнению

$$
\bar{u}^{\prime \prime}+b\|u\|_{p, \Omega}^{p} \bar{u}^{\prime}+\frac{12 a^{2}}{l^{2}} \bar{u}=\frac{6 a^{2}}{l}\left(\psi_{1}+\psi_{2}\right) .
$$

Начальные условия, необходимые для его интегрирования, получаются из условий (3):

$$
\bar{u}(0)=\int_{\Omega} u(x, 0) d x=0, \quad \bar{u}^{\prime}(0)=\int_{\Omega} u_{t}(x, 0) d x=0 .
$$

Для решения полученной задачи выберем в (11) верхнюю границу неравенства, что позволяет сделать замену

$$
\|u\|_{p, \Omega}^{p}=K(t)
$$

приводящую (15) к линейному уравнению

$$
\bar{u}^{\prime \prime}+b K(t) \bar{u}^{\prime}+\frac{12 a^{2}}{l^{2}} \bar{u}=F_{2}(t)
$$

с правой частью

$$
F_{2}(t)=\frac{6 a^{2}}{l}\left(\psi_{1}+\psi_{2}\right)
$$

Как известно, единственное решение задачи (18), (16) существует при $t \in(0, T)$ для непрерывных функций $K(t)$ и $F_{2}(t)$. После его подстановки вместе с (17) в формулу (13) будет найдена функция

$$
u(x, t)=\frac{3 x}{l}\left(\frac{x}{l}-1\right)\left(\psi_{1}+\psi_{2}-\frac{2}{l} \bar{u}\right)+x \frac{\psi_{2}-\psi_{1}}{l}+\psi_{1},
$$

которую примем за приближенное решение как задачи (2)-(4), так и аппроксимируемой ею задачи (1), (3), (4).

\section{3. Пример}

Будем искать решение задачи (2)-(4), рассматривая (2) как аппроксимирующие уравнение для (1). Уравнение вида (1) моделирует, в частности, некоторые нестационарные гидродинамические процессы в трубах, при этом $u-$ скорость течения жидкости, $a-$ скорость звука в жидкости, для воды $a \approx 1450 \mathrm{~m} / \mathrm{c}, b$ зависит 
от физических характеристик трубы и в некоторых случаях может быть принята равной $1 / 3$.

Пусть $p=3$. Положим $l=1$ и выберем граничные условия (4) в виде $\psi_{1}(t)=\psi_{2}(t)=t$, тогда в (15) правая часть $F_{2}(t)=12 a^{2} t$, а величина $F(T)$, задаваемая формулой (10), определяется как

$$
F(T)=3 C_{1} C_{2} T^{2}+2 a^{2} C_{3} T^{4} .
$$

При этом функция (19) запишется как

$$
u(x, t)=6 x(x-1)(t-\bar{u})+t .
$$

Перейдем к определению постоянных, входящих в эти выражения. В силу того, что $\Omega=[0,1]$, можно принять $C_{1}=C_{2}=1$. Из (12) следует условие положительности функции $K(t): b F(T) t<2$, т.е.

$$
t<\frac{2}{b\left(3 T^{2}+2 a^{2} C_{3} T^{4}\right)} .
$$

Учитывая, что $t \leqslant T$, перейдем к неравенству

$$
C_{3} \geqslant \frac{2-3 T^{2} b T}{2 a^{2} b T T^{4}}
$$

из которого в силу неотрицательности $C_{3}$ следует, что $T^{3} \leqslant 2 /(3 b)$. Примем $T^{3}=1 /(3 b)$, тогда можно положить

$$
C_{3}=\frac{3 \sqrt[3]{9 b^{2}}}{2 a^{2}}
$$

При выбранном выше значении $b$ получаем $T=1$. Находя последовательно $C_{3}$, $F(T), K(t)$ и возвращаясь к (18), приходим к задаче

$$
\begin{gathered}
\bar{u}^{\prime \prime}+\frac{2}{1-t} \bar{u}^{\prime}+12 a^{2} \bar{u}=12 a^{2} t, \\
\bar{u}(0)=0, \quad \bar{u}^{\prime}(0)=0 .
\end{gathered}
$$

Ее приближенное решение с учетом только значимых слагаемых записывается в виде

$$
\bar{u}(t) \approx t+(t-1)(0,696 \cos (2900 \sqrt{3} t)-\cos (2900 \sqrt{3}(t-1))-\sin (2900 \sqrt{3}(t-1))) .
$$

Подстановка найденной функции в (20) дает приближенное решение задачи $(2)-(4)$ :

$$
\begin{aligned}
u(x, t) \approx 6 x(x-1)[\cos (2900 \sqrt{3}(t-1))+ & \sin (2900 \sqrt{3}(t-1))- \\
& -0,696(t-1) \cos (2900 \sqrt{3} t)]+t .
\end{aligned}
$$

Так как уравнение (2) является аппроксимирующим по отношению к уравнению (1) и его интегрирование проводится при тех же условиях (3), (4), что и для 
уравнения (1), то функцию (21) будем считать приближенным решением задачи (1), (3), (4).

\section{Заключение}

В работе предложен приближенно-аналитический метод нахождения решения задачи (2)-(4), состоящий, во-первых, в переходе от исходного нагруженного уравнения (2) к ассоциированному с ним обыкновенному дифференциальному уравнению (15), а во-вторых, в линеаризации (15) с помощью априорной оценки решения исходной задачи вида (11). Полученное приближенное решение выражается аналитически функцией (21). Данный метод применим к нагруженным дифференциальным уравнениям в частных производных, содержащим интеграл по пространственной переменной от $p$-й степени неизвестной функции при некоторых допущениях относительно $p$. Основную сложность в реализации метода представляют процедуры установления априорной оценки (11) и подбора констант, входящих в это и другие необходимые неравенства.

\section{Список литературы}

[1] Лионс Ж.-Л. Некоторые методы решения нелинейных краевых задач. М: Едиториал УРСС, 2010. 586 с.

[2] Medeiros L.A. On the weak solutions of nonlinear partial differential equations // Anais da Academia Brasileira de Ciencias. 1981. Vol. 53, № 1. Pp. 13-15.

[3] Lourêdo A.T., Ferreira de Araújo M.A., Miranda M. M. On a nonlinear wave equation with boundary damping // Mathematical Methods in the Applied Sciences. 2014. Vol. 37, № 9. Pp. 1278-1302.

[4] Бозиев О.Л. Решение начально-краевой задачи для нелинейного гиперболического уравнения с помощью двойной редукции к нагруженным уравнениям // Известия Кабардино-Балкарского научного центра РАН. 2014. № 4(60). C. $7-12$.

[5] Бозиев О.Л. Применение нагруженных уравнений к приближенному решению дифференциальных уравнений в частных производных со степенной нелинейностью // Вестник ТвГУ. Серия: Прикладная математика. 2015. № 1. С. 127136.

[6] Бозиев О.Л. Приближенное решение нагруженного гиперболического уравнения с однородными краевыми условиями // Вестник Южно-Уральского государственного университета. Серия: Математика. Механика. Физика. 2016. Т. 8, № 2. C. 14-18.

[7] Демидович Б.П. Лекции по математической теории устойчивости. М.: Наука, 1967. 472 с. 


\section{Библиографическая ссылка}

Бозиев О.Л. Приближенное решение нагруженного гиперболического уравнения с однородными начальными условиями // Вестник ТвГУ. Серия: Прикладная математика. 2017. № 2. С. 49-58.

\section{Сведения об авторах}

\section{1. Бозиев Олег Людинович}

старший научный сотрудник отдела Автоматизации и информатизации региональных систем управления Института информатики и проблем регионального управления Кабардино-Балкарского научного центра РАН.

Россия, 360000, г. Налъчик, ул. И. Арманд, 37А. E-mail: boziev@yandex.ru. 


\title{
AN APPROXIMATE SOLUTION OF LOADED HYPERBOLIC EQUATION WITH HOMOGENIOS INITIAL CONDITIONS
}

\author{
Boziev Oleg Ludinovich \\ Institute of Computer Science and Problems of Regional Management of KBSC, \\ Russian Academy of Sciences \\ Russia, 360000, Nalchik, I. Armand str., 37A. \\ E-mail: boziev@yandex.ru
}

\begin{abstract}
Received 04.02.2017, revised 31.03.2017.
The article offers a method for solving a mixed problem with homogeneous initial conditions for a loaded hyperbolic equation with integral natural degree module of unknown function. An approximate solution is sought by means of a priori estimates of the solution of the problem. We obtained a formula expressing the solution through the solution of the ordinary differential equation associated with the source loaded equation.
\end{abstract}

Keywords: nonlinear partial differential equations, loaded partial differential equations, a priori estimates, approximate solutions.

\section{Bibliographic citation}

Boziev O.L. An approximate solution of loaded hyperbolic equation with homogenios initial conditions. Vestnik TvGU. Seriya: Prikladnaya Matematika [Herald of Tver State University. Series: Applied Mathematics], 2017, no. 2, pp. 49-58. (in Russian)

\section{References}

[1] Lions J.L. Nekotorye Metody Reshenia Nelineinyh Kraevyh Zadach. [Some Methods for Solving of Nonlinear Boundary Value Problems]. Moscow, Editorial URSS Publ., 2010. 586 p. (in Russian)

[2] Medeiros L.A. On the weak solutions of nonlinear partial differential equations. Anais da Academia Brasileira de Ciencias, 1981, vol. 53(1), pp. 13-15.

[3] Lourêdo A.T., Ferreira de Araújo M.A., Miranda M.M. On a nonlinear wave equation with boundary damping. Mathematical Methods in the Applied Sciences, 2014, vol. 37(9), pp. 1278-1302.

[4] Boziev O.L. Solving an initial-boundary value problem for the nonlinear hyperbolic equation using a double reduction to the loaded equations. Izvestiya KabardinoBalkarskogo Nauchnogo Tsentra RAN, 2014, no. 4, pp. 7-13. (in Russian) 
[5] Boziev O.L. Application of loaded equations to approximate solutions of partial differential equations with the power nonlinearity. Vestnik TvGU. Seriya: Prikladnaya matematika [Herald of Tver State University. Series: Applied Mathematics], 2015, no. 1, pp. 127-136. (in Russian)

[6] Boziev O.L. An approximate solution of loaded hyperbolic equation with homogenios boundary conditions. Bulletin of the South Ural State University. Ser. Mathematics. Mechanics. Phisics, 2016, vol. 8(2), pp. 14-18. (in Russian)

[7] Demidovich B.P. Lektsii po Matematicheskoy Teorii Ustoichivosti [Lectures on Mathematical Theory of Stability]. Moscow, Nauka Publ., 1967. 472 pp. (in Russian) 